\title{
Bitcoin and Stock Markets: Are They Connected? Evidence from Asean Emerging Economies
}

\section{Abdollah Ah Mand ( $\square$ abdollaha@sunway.edu.my )}

Sunway University

Hassanudin Mohd Thas Thaker

Sunway University

\section{Research}

Keywords: Cryptocurrencies, bitcoin, stock market, emerging economies

Posted Date: May 12th, 2020

DOI: https://doi.org/10.21203/rs.3.rs-25524/v1

License: (c) (i) This work is licensed under a Creative Commons Attribution 4.0 International License. Read Full License 


\title{
TITLE PAGE
}

\section{Bitcoin and Stock Markets. Are they Connected? Evidence from Asean Emerging Economies}

\author{
Abdollah Ah Mand*1, Hassanudin Mohd Thas Thaker2 \\ Email of corresponding author: abdollaha@sunway.edu.my \\ 1,2 Sunway University, Malaysia
}

\begin{abstract}
\end{abstract}

\section{Background:}

Cryptocurrencies, especially Bitcoin, has become popular for investors in recent years. The volatility of bitcoin and time horizon are the center point for investment decisions. However, attention is not often drawn to the relationship between bitcoin and equity indices. This study investigates the volatility and time frequency domain of bitcoin among five Asean countries through a rich database which covers daily data from July 2010 until April 2019.

\section{Methods:}

Advanced econometrics and Wavelets Cross-Coherence Spectrograms, this study investigates the existence of long run association between bitcoin and the studied market indices. M-GARCH analysis is been employed to investigate the unconditional volatility of market indices and Bitcoin.

\section{Results:}

The findings present the long run association with positive (Philippines) and negative (Japan, Korea, Singapore, Hong Kong) relations. Moreover, only one market (KOREA) shows a short run association with bitcoin. The MGARCH analysis reveals, most of the selected Asean countries have a low unconditional volatility with bitcoin. Except for Philippines in which the co-movement is average, Wavelet analysis reveals the presence of a strong and long comovements for most of the selected Asean countries with bitcoin.

\section{Conclusions:}

Most of our results are consistent and illustrate different dimensions of long and short run relationship, volatilities, correlations, and time-frequency analysis. This study utilized Asean emerging economies which are rarely available in the literature as existing studies are more skewed towards the West. We believe the outcomes of this study will be a significant for industry practitioners (i.e., retail and institutional investors) on designing better strategies to diversify the stock portfolio with different holding period horizons and dimensions.

\section{Authors:}

*Dr Abdollah Ah Mand (Corresponding Author), Sunway University, Malaysia. (Email: abdollaha@sunway.edu.my)

ORCID: http://orcid.org/0000-0002-8778-3102

Dr Hassanudin Mohd Thas Thaker, Sunway University, Malaysia. (Email: hassanudint@sunway.edu.my)

ORCID: http://orcid.org/0000-0002-9182-9045 


\title{
Bitcoin and Stock Markets. Are they Connected? Evidence from Asean Emerging Economies
}

\begin{abstract}
Background:

Cryptocurrencies, especially Bitcoin, has become popular for investors in recent years. The volatility of bitcoin and time horizon are the center point for investment decisions. However, attention is not often drawn to the relationship between bitcoin and equity indices. This study investigates the volatility and time frequency domain of bitcoin among five Asean countries through a rich database which covers daily data from July 2010 until April 2019.

Methods:

Advanced econometrics and Wavelets Cross-Coherence Spectrograms, this study investigates the existence of long run association between bitcoin and the studied market indices. M-GARCH analysis is been employed to investigate the unconditional volatility of market indices and Bitcoin.

Results:

The findings present the long run association with positive (Philippines) and negative (Japan, Korea, Singapore, Hong Kong) relations. Moreover, only one market (KOREA) shows a short run association with bitcoin. The MGARCH analysis reveals, most of the selected Asean countries have a low unconditional volatility with bitcoin. Except for Philippines in which the co-movement is average, Wavelet analysis reveals the presence of a strong and long comovements for most of the selected Asean countries with bitcoin.

\section{Conclusions:}

Most of our results are consistent and illustrate different dimensions of long and short run relationship, volatilities, correlations, and time-frequency analysis. This study utilized Asean emerging economies which are rarely available in the literature as existing studies are more skewed towards the West. We believe the outcomes of this study will be a significant for industry practitioners (i.e., retail and institutional investors) on designing better strategies to diversify the stock portfolio with different holding period horizons and dimensions.
\end{abstract}

\section{Background}

Bitcoin as an investment asset (see Baur, Hong, \& Adrian, 2017; Bouri, Molnár, Azzi, Roubaud, \& Hagfors,2017a), has been the center point of attention for investors, international participants, regulators, and media after its introduction by Nakamoto (2008). It has become the largest cryptocurrency market of today and the first decentralized digital currency, retaining its position as leader of the cryptocurrency market (Shaen Corbet et al., 2018). This common interest among investors can be derived from its innovativeness, straightforwardness, and increasing popularity (Urquhart, 2016). Its uniqueness as peer to peer electronic cash systems makes it more attractive for investors. This electronic system allows direct online transactions from one party to another, eliminating the time-consuming and complex cash systems of financial institutions. Moreover, unlike many other financial assets, the online system of bitcoin has no connotation with any sophisticated authority and has no physical representation which is substantially distinguishable. Furthermore, the algorithm on which all transactions can be traced with lower transaction costs and government-free structure makes bitcoin more popular and places the cryptocurrency in mainstream news. These points on the popularity of bitcoin are one of the contributors to the rise in the pricing of Bitcoin. Albeit the opportunities in bitcoin, it nevertheless carries big challenges as well.

Although cryptocurrency investors enjoy several benefits such as efficient and advanced payment systems, there are risks that could be destructive to consumers, businesses, financial systems, and national security. Thus, there have been mixed views on cryptocurrencies and their future continues to be the major reassurance of their excessive volatility and market values. Exploring this fact is one of the interests of this research which seeks to determine the short and long run volatility of bitcoin in the complex world of 
cryptocurrencies which remains unclear for most market participants and scientists. Furthermore, the relationship between bitcoin and the equity markets is a new concern on which this study is looking into. This is inspired by studies conducted by Baur et al. (2017) and Bouri et al. (2017) which investigate the relationship of several financial assets, including the more conventional investments such as international equities, bonds, and currencies, as well as commodities which can provide useful implications for investors and policy makers. In examining the equity market, we consider a global equity index and pay attention to significant equity markets with major bitcoin mining in their regions such as Japan, Korea, Singapore, Philippines, and Hong Kong. These equity markets are significant for investors in Asia. To find more transparent results, this research excludes the Chinese equity market even though Chinese users are an important group of stakeholders in the Bitcoin market, as the People's Bank of China banned the use of bitcoin (see Shuyue Yi et al., 2018). In this regard, our study looks at the long run and short run relationship between bitcoin and five major equity markets separately and tries to answer the causal relationship among those equity markets and bitcoin in both short and long run. Our final intention in this study is to understand which time horizon is the ideal time to invest in bitcoin. In doing this, investors may choose to invest in short, medium, and long-term investments on bitcoin. This question opens new windows to bitcoin investors and provides clearer time horizons, enabling them to identify the most suitable time to invest on bitcoin. Such is deemed important since the study on time horizon and bitcoin investment has been largely absent in the literature.

Moreover, we must enhance our partial knowledge of bitcoin's market integration with other financial assets for several other reasons. First, it influences the structure and implementation of policies to continue financial stability. Second, it stimulates the conclusions of policy makers in nations that are likely to consider bitcoin as an official digital currency or as portion of their foreign reserves. Third, from asset allocation and risk management perspectives, it has significant influence on investors. As mentioned earlier, studies have been limited in the literature on the relationship between bitcoin and other economic assets which mostly relies on unconditional correlations. However, in the present study, we offer a broad view of the long and short run relationships between bitcoin and global equity markets as well as the volatility and time horizon of bitcoin investment.

Our research contribution arises from three main aspects. First, the application of the long run and short run causality and VECM approach allows us to map the long run and short run relationship of five major equity markets. The discussion concerning the relationship between bitcoin (the largest cryptocurrency) and five important equity markets provides new empirical evidence under different market situations. Second, our findings add to the literature on volatility of cryptocurrencies with respect to bitcoin by employing a rich data and GARCH approach. Based on the existing literature, further development on the volatility of bitcoin is essential due to the inconclusive findings of different volatility models and differences in the nature of data. Hence, from the perspective of volatility, there are scopes to be discovered. Lastly, our findings uncover a significant question on the time horizon of investment on bitcoin. Such findings extend our limited understanding of bitcoin incorporation by revealing a time varying nature of equity markets that seems to challenge the general view in the current empirical literature in which bitcoin is isolated from the global financial system. The analysis in this paper is divided into three parts. Firstly, we investigate the volatility behavior of bitcoin in comparison to five different equity market indices. According to Blau (2017), the high volatility of bitcoin until 2014 is not related to speculative trading. This is in contradiction to Cheah and Fry (2015) and Cheung, Roca, and Su (2015), who find bitcoin to build speculative bubbles in the same time frame. In the present study, we aim to investigate the volatility of bitcoin in the short run and long run by employing Multivariate Generalized Autoregressive Conditional Heteroscedasticity (M-GARCH) to further understand the volatility of bitcoin by using a database which covers the daily prices of bitcoin until the end of first quarter of 2019. Secondly, this paper investigates the long run and short run relationship of bitcoin with five major equity indices by using time series analysis of VECM and Granger causality test. Lastly, time frequency domain analysis is conducted by employing 
Wavelet Transform (CWT) to determine the suitable time (short, medium, and long term) to invest in different equity indices.

\section{Literature Review}

There is an ever-growing literature examining bitcoin. In an influential work, Dwyer (2015) shows that the average monthly volatility of bitcoin is higher than that of gold or a set of foreign currencies while Urquhart (2016) discloses the inefficient returns of bitcoins, which is also supported by Nadarajah and Chu (2017) and Bariviera (2017). Bitcoin is an electronic system that eases the allocation of funds between parties. Based on peer-to-peer networking and algorithm, it allows users to make nameless transactions, just like cash, but through the Internet and without the need for financial mediators. In this sense, bitcoin is fully decentralized without the intervention of third parties, such as central banks or government financial agencies (Weber, 2014). In recent time, bitcoin is subject to various studies. For instance, Kastiama (2017) investigated the volatility of bitcoin by implementing different GARCH-type models of volatility which showed the importance of having both short-run and long-run components of the conditional variance. The research concluded that the AR-CGARCH was the most suitable model to study the volatility of bitcoin. However, this study is challenged by a recent study by Amélie Charles and Olivier Darné (2019) which claims the absence of a conclusive result for the volatility model of bitcoin. Furthermore, Conrad et al. (2018) examined the drivers of long-term volatility of bitcoin and compared them to other asset classes, e.g. gold. The authors found that bitcoin volatility was distinct compared to other asset classes. Also, researchers like Bariviera (2017) and Nadarajah and Chu (2017) confirmed the inefficiency of bitcoin. Moreover, Guesmi et al. (2018) examined the joint dynamics of bitcoin and different financial assets via a multivariate GARCH model. Existing arguments highlight the need for further investigations on this area. Nevertheless, a question yet to be answered in the literature is the relationship between bitcoin and equity markets. This relationship has received little attention in the existing literature. Cryptocurrencies are considered a new era of research and still need to be investigated according to different perspectives.

Bitcoin and its price dynamics has been the subject of various studies. For instance, Urquhart (2017) shows the significant clustering of bitcoin prices. Shen, Urquhart and Wang (2019) found the significant relationship between the trading volume of bitcoin and its number of tweets. However, there is another body of literature on the relationship between other financial assets to bitcoin. For instance, Dyhrberg (2016) looked into the hedging possibilities of bitcoin, the US dollar and UK stock market and found a similar capability of hedging to gold. Additionally, Bouri et al. (2017a) examined the relationship between gold, global uncertainty and bitcoin via a quantile regression approach and found that bitcoin has the capability to be hedged against global uncertainty for short investment horizons and in the bull market regimes. However, in a later study Bouri et al. (2017b), the researchers found limited evidence of hedging capability and safe heaven properties for bitcoin. Following their study, Guesmi et al. (2018) made evident that bitcoin has some hedging capabilities and diversification benefits against many other safe heaven assets. However, Klein, Thu, and Walther (2017) conclude that bitcoin offers no hedging capabilities like gold which also holds for the brad cryptocurrency index CRIX. On the other hand, Urquhart and Zhang (2019) argue that bitcoin can be a hedge at an intraday level for the CHF, EUR and GBP currencies. Hence, the existing literature has presented an inconclusive finding on the capability of bitcoin hedging against safe heaven assets. However, a substantial majority of published papers on the economics of bitcoin address the issue from an empirical perspective without addressing the relationship between bitcoin and equity market indexes, as well as the short run and long run causality of such relationships (if exist). These are factors yet to be explored clearly in need of discovery.

Ever since the invention of bitcoin, investors have been concerned about adding bitcoin to their portfolio of investment. As such, there exists a number of studies which investigated bitcoin from the portfolio perspective. However, Platanakis and Urquhart (2018) warn over the risks of having cryptocurrencies in the portfolio which should not be ignored in the decision-making process. In their study, 
Brière et al. (2015) investigated the performance of portfolio with bitcoin as part of the portfolio. They employed Sharpe ratio of diversified portfolio and found that bitcoin improves the performance ratio. Also, they found the benefits of diversification by conducting stock-bond portfolio. The fact that bitcoin is isolated from other financial and economic variables makes it an important diversifier. Nevertheless, the introduction of bitcoin lines funded by global investment banks enhances accessibility to the bitcoin market. In particular, the launch of future contracts based on bitcoin prices in 2017 increased the legality of bitcoin as an investment and moved it closer to the main screen for financial world. Hence, these developments give the signal that bitcoin should not be isolated by the investment communities (Polasik et al., 2015). Studies by Bouri et al. (2017a) and Bouri et al. (2017b) on bitcoin's ability to diversify, especially during bear market, reveal the network construction between bitcoin and equity indices. In their findings, there is a poor relationship between bitcoin and equities and this relationship is not steady over time and is precious with structural breaks. This weak relation between bitcoin and other financial assets may be due to bitcoin not sharing many common price determinants with those financial assets (Bouoiyour, Selmi, Tiwari, \& Olayeni, 2016; Kristoufek, 2013).

In this study, we extend the above-mentioned literature by discovering the long run and short run relationship between bitcoin and a set of global and country equity indexes. Furthermore, the volatility of bitcoin for the short and long run is examined by using an approach that is different than the existing literature on bitcoin's volatility, as this study employs a rich sample from the beginning of bitcoin until the present. This paper also considers the time horizon of bitcoin investment from three different horizons of short term, medium, and large terms by using an advanced methodology which makes this paper different from the existing studies on bitcoin.

\section{Methodology}

\subsection{Data Collection}

We use daily series data from 20th July 2010 until 26th April 2019. The data on bitcoin and Asean stock indexes was collected from (https://www.investing.com/) and (https://www.wsj.com/marketdata/quotes/index). We furnish the information with regard to the data based on country basis as well as

$\mathrm{BC}$ and market index. We further convert the index values using the formula of $R=\ln \left[\frac{V i_{t}-V i_{t-1}}{V i_{t-1}}\right]$ where $R$ represents return for BC and equity indexes, $V i$ is current index value, $V i_{t-l}$ is the previous value, and $l n$ represents natural log. Our data is rich in terms of its total number of observations and captures divergence in volatilities and correlation of BC and Asean markets due to 2013's April meltdown, the Fammed 2013 Bubble, the bankruptcy of Mt. Gox Bitcoin Exchange, the summer sale of 2017, and China's Stern intervention. We excluded some years given the fact of missing data in stock indexes during the analysis period. We opted five Asean emerging economies as these countries show tremendous growth in different types of cryptocurrencies and recorded good momentum in bitcoin and other assets. The details of indexes used are shown in the table below:

\section{[Insert Table 1 here]}

This paper adopts multiple techniques to investigate the relationship between bitcoin and equity market returns in selected developed and developing countries. The approached methodology follows the traditional econometric method of VECM in combination with the Time-Frequency domain analysis via Wavelet techniques. VECM restricts the long run behavior of the endogenous variable to cointegrate while allowing short run adjustment. The cointegrating term is known as an error correction term, since the deviation from long-run equilibrium is corrected gradually through a series of partial short-run adjustments. In this research, VECM approach examines the equilibrium between the price of bitcoin index and equity market indices for five selected countries. We also employ the Multivariate Generalized Autoregressive Conditional Heteroscedasticity (M-GARCH) to identify the changes in correlation and volatilities of bitcoin 
and Asean stock markets indices over time together with its directions (positive or negative) and magnitude (stronger or weaker). Following that, we also analyse the time frequency domain using Wavelet investigation and balance its findings through cross-correlation and cross-coherence analysis. Initially, it expresses how the cross-correlation between the two series varies across multiple scales, followed by the time-based evolution of the co-movement between the series along with directional leadership. Thus, the cross-coherence analysis helps us unravel new evidence on whether the higher price of bitcoin is influencing the prices of selected equity markets or the opposite, for instance, higher equity market prices are happening as a result of increased bitcoin price. Moreover, since Wavelet analysis operates in a time-frequency domain (i.e., time horizon), the significance of the hypothesized interactions and the magnitudes of interactions are also discoverable. In the following sections, we explain in detail the estimations of the three tools.

\subsection{Long Run \& Short Run Associations Using Time Series Analysis (VECM \& Granger Causality)}

Vector Error Correction Model (VECM), also called restricted VAR, is designed to use non-stationary variables that are known to be cointegrated. VECM restricts the long run behavior of the endogenous variables to converge to their cointegrating relationship while permitting for short-run dynamic adjustment. VECM distinguishes short- and long-term associations in a model. In this content, the lagged structure of error term refers to short term deviation from long term equilibrium. The VECM also enables for Granger causality relations and Error Correction Term. The Granger causality test is also implemented in this research to understand the relationship of the dependent variables and explanatory variable. The general equation for VECM is presented as follows:

$\Delta_{x t}=\alpha_{t}+\Omega X_{t-1}+\sum_{t=1}^{k=1} \gamma_{t} \Delta_{t-1}+\varepsilon_{t}$

Where: $\mathrm{X}_{\mathrm{t}}$ is used to denote the chosen endogenous variables for each model and $\varepsilon_{\mathrm{t}}$ is disturbance error term. From the above equation, the VECM model for this study can be illustrated as the equation below:

For market index (MI) for each country (i= Japan, Korea, Singapore, Philippines, and Hong Kong):

$$
\Delta M I_{i}=\mu_{1 t}+\theta_{1}\left(\Delta M I-\gamma_{0}-\gamma_{1} B C_{i}\right)_{t-1}+\sum_{i=1}^{j} \beta_{1, j} M I_{1 t-i}+\sum_{i=1}^{j} \beta_{2, j} B C_{1 t-j}+\varepsilon_{1 t}
$$

Where: $\mathrm{MI}$ is market index and $\mathrm{BC}$ refers to $\mathrm{BC}$ price index (bitcoin) which is the ratio of price and standard deviation of bitcoin. Causality test is implemented to understand the direction of the relationship between variables. In regression modeling, the underlying theory will indicate the direction of causality between $\mathrm{Y}$ and $\mathrm{X}$, which is in the context of single equation models, is generally from $\mathrm{X}$ to Y. Hence, to understand this relationship and its directions, this research applies the Granger causality test.

\subsection{Multivariate Generalized Autoregressive Conditional Heteroscedasticity (M-GARCH)}

Multivariate GARCH-DCC is the modified version of Bollerslev's (1990) constant conditional correlation (CCC-GARCH) as introduced by Engle and Kevin (2001) and Engle (2002). This research employs MGARCH-DCC to explore two important phenomena; first, to investigate the fluctuations in correlation and volatilities of bitcoin return and Asean stock market indices over time. Second, to determine the directions and magnitude of existing correlations. MGARCH-DCC eases the assumption of CCC-GARCH on the conditional correlations of series as persistent following the assumption of having positive amount for the time dependent conditional correlation matrix. This assumption is impractical in various empirical 
investigations. In the process of employing MGARCH-DCC, two stages were observed; first, the estimation of standard deviation (SD) from univariate GARCH, and second, using SD to calculate the standardized residuals and correlation matrix. By referring to Engle (2002), the equation for Multivariate conditional covariance matrix $\left(H_{t}\right)$ for this research can be written as follows:

$H_{t}=D_{t} R_{t} D_{t}$

where $D_{t}=$ diagonal matrix of conditional time varying, standardized residuals $\left(\varepsilon_{t}\right)$ that are obtained from the univariate GARCH models (on-diagonal elements), and $R_{t}=$ time varying correlation matrix (offdiagonal elements).

The log-likelihood of the above estimator can be written as follows:

$$
\begin{aligned}
L & =-\frac{1}{2} \sum_{t=1}^{T}\left(k \log (2 \pi)+2 \log \left|H_{t}\right|+r_{t} H_{t}^{-1} r_{t}\right) \\
& =-\frac{1}{2} \sum_{t=1}^{T}\left(k \log (2 \pi)+2 \log \left|D_{t} R_{t} D_{t}\right|+r_{t} D_{t}^{-1} R_{t}^{-1} D_{t}^{-1} r_{t}\right. \\
& =-\frac{1}{2} \sum_{t=1}^{T}\left(k \log (2 \pi)+2 \log \left|D_{t}\right|+\log \left(\left|R_{t}\right|+\varepsilon_{t} R_{t}^{-1} \varepsilon_{t}\right)\right.
\end{aligned}
$$

here, $\varepsilon_{t} \sim N\left(0, R_{t}\right)$ are the standardized residuals $\left(\varepsilon_{t}\right)$ of their conditional standard deviations.

The conditional variances for any individual asset can be obtained from the univariate GARCH model as follows:

$h_{i t}=\omega_{i}+\sum_{p=1}^{P i} \alpha_{i p} r_{i t-p}^{2}+\sum_{q=1}^{Q 1} \beta_{i q} h_{i t-p}$ for $i=1,2,3, \ldots . k$

where $\omega_{i}, \alpha_{i p}$ and $\beta_{i q}$ are non-negative and $\sum_{p=1}^{P_{i}} \alpha_{i p}+\sum_{q=1}^{Q_{i}} \beta_{i q}<1 . h_{i t}$ is the estimated conditional variance of individual asset, $\alpha_{i p}$ is the short-run persistence of shocks to return P (the ARCH effects) and $\beta_{i q}$ is the contribution of shocks to return $\mathrm{Q}$ to long-run persistence (the GARCH effects).

Having obtained the conditional variances for any individual asset, the dynamic correlation structure can thus be written as follows:

$$
\begin{gathered}
Q=\left(1-\sum_{m=1}^{M} \alpha_{m}-\sum_{n=1}^{N} \beta_{n}\right) \bar{Q}+\sum_{m-1}^{M} \alpha_{m}\left(\varepsilon_{t-m} \varepsilon_{t-m}\right)+\sum_{n-1}^{N} \beta_{n} Q_{t-n} \\
R_{t}=Q_{t}^{*-1} Q_{t} Q_{t}^{*-1}
\end{gathered}
$$

where, $\bar{Q}$ is the unconditional covariance of the standardized residuals $\left(\varepsilon_{t}\right)$; and $\mathrm{Q}^{*}$ is a diagonal matrix composed of the square root of the diagonal elements of $Q_{t}$, which is as follows:

$Q_{t}^{*}=\left[\begin{array}{cccc}\sqrt{q_{11}} & 0 & \ldots & 0 \\ 0 & \sqrt{q_{22}} & \cdots & 0 \\ \vdots & \vdots & \vdots & 0 \\ 0 & 0 & \cdots & \sqrt{q_{k k}}\end{array}\right]$ 
The component of $R_{t}$ will be $P_{i j t}=\frac{q i j t}{\sqrt{q i i q j j}}$ and the matrix $R_{t}$ will be positive definite/constant. The $\mathrm{K}$ is covariance $H t$ is positive definite/constant and can be written as $H_{t}=D_{t} R_{t} D_{t}$. The breakdown of $H_{t}$ allows separate specification of the conditional volatilities and conditional correlations. For instance, one can utilize the $\operatorname{GARCH}(1,1)$ model for the variance $\sigma_{i, t-1}^{2}$, namely.

$V\left(r_{i t} \mid \Omega_{t-1}\right)=\sigma_{i, t-1}^{2}=\bar{\sigma}_{i}^{2}\left(1-\lambda_{1 i}-\lambda_{2 i}\right)+\lambda_{1 i} \sigma_{i, t-2}^{2}+\lambda_{2 i} r_{i, t-1}^{2}$

where, $\bar{\sigma}_{i}^{2}$ is the unconditional variance of the asset return; $\lambda_{1}$ and $\lambda_{2}$ are individual asset volatility parameters. Under the constraint $\lambda_{1 \mathrm{i}}+\lambda_{2 \mathrm{i}}=1$, the unconditional variance $\left(\bar{\sigma}_{i}^{2}\right)$ will be extinct in the above equation. Hence, the Integrated GARCH (I-GARCH) model will be estimated on which discloses that any shocks to the variance are permanent and non-stationarity of the conditional variance.

\subsection{Continuous Wavelet Transform (CWT)}

Researches in finance and economics are witnessing the growth on the usage of wavelet transformation analysis. There are some points on the aspects of wavelet methodology that suit the model of the current study. By implementing wavelet analysis, researchers are able to fetch high quality information confined into a signal in various scales. Since the VECM analysis of this research employs the traditional econometric testing for short and long run relationship between bitcoin and equity market indices, wavelet testing is placed exclusively to not only perform the robustness check on VECM results, but to also explore new visions in the time-frequency domain by using cross-correlation and coherency analysis.

Using a wavelet transform requires a restricted waveform expressed as $\Psi(t) \mathrm{W}(\mathrm{t})$; called commonly a mother wavelet. While the mother wavelet integrates to zero, its harmonizing normalized counterpart $\phi$ integrates to 1 and is known as a father wavelet. The former is used for interrogating signals in greater details and is therefore more relevant for higher frequency testing. As such, we go on to build a wavelet function by forging a series of plans including mother and father wavelets. We achieve this by transformation and scaling, expressed mathematically as:

$$
\begin{aligned}
\psi_{s, k}(t) & =\frac{\psi\left(\frac{t}{2^{s}}-k\right)}{2^{\frac{j}{2}}} \\
\phi_{s, k}(t) & =\frac{\phi\left(\frac{t^{s}}{2^{s}}-k\right)}{2^{\frac{s}{2}}}
\end{aligned}
$$

In the two equations above, $\mathrm{s}=1, \ldots, \mathrm{S}$, where $\mathrm{S}$ and $\mathrm{k}$ are scaling and translation parameters respectively. Also, the wavelet transformation of a signal can be represented as:

$y(t)=\sum_{k} \theta_{s, k} \phi_{s, k}(t)+\sum_{k} d_{s, k} \psi_{s, k}(t)+\sum_{k} d_{s-1, k} \psi_{s-1, k}(t)+\ldots+\sum_{k} d_{1, k} \phi_{1, k}(t)$

the smooth coefficient is $\theta_{s, k}=\int y(t) \phi_{s, k}(t) d t$ and detail coefficient is $d_{s, k}=\int y(t) \psi_{s, k}(t) d t$. Together $\theta_{s, k}$ and $d_{s, k}$ represent how much a particular wavelet function contributes to the overall signal. Following the methodology of Rua and Nunes (2009) and Kristoufek (2013), we have the wavelet Morlet as following;

$\psi_{u, s}(t)=\frac{1}{\sqrt{s}} \psi\left(\frac{t-u}{s}\right), \varphi(.) L^{2} \subset \mathbb{R}$

At this stage, the cross-wavelet spectrum of bitcoin returns and equity market indices for individual equity market series are as follow;

$W_{\text {Equity indices, Returns }}^{1}(\tau, s)=W_{\text {indices }}(\tau, s) W_{\text {return }}^{*}(\tau, s)$

Accordingly, the cross-wavelet spectrum's cross-coherency is achieved by taking the absolute value of the squares of the smoothed spectrums, as below: 
$C C_{(\tau, s)}^{2}=\frac{\mid S\left(\left.s^{-1} W_{x, y}(\tau, s)\right|^{2}\right.}{S\left(s^{-1}\left|W_{x}(\tau, s)\right|^{2}\right) S\left(s^{-1}\left|W_{y}(\tau, s)\right|^{2}\right)}$

Eq. (14) is useful for capturing the degree to which two time-series co-move across time and frequencies, an improvement over Fourier transforms which deal with frequencies alone. A $C C_{(\tau, s)}^{2}$ value ranges from 0 to 1 , where 0 indicates no co-movement and 1 as perfect strong co-movement.

\section{Empirical Results and Discussion}

\subsection{Descriptive Statistics}

Table 2 provides a description of the data used for analysis. The data is described based on mean, maximum, minimum and standard deviation. In terms of mean values, STI index recorded higher mean value followed by BC, JPN, PHIL, HK and KOR. In terms of maximum values, BC obtained higher value as compared to others. The same goes for minimum value as well in which $\mathrm{BC}$ recorded minimum values. We can observe that large dispersion occurs at BC market where the standard deviation is of 0.070157 followed by JPN, HK, PPHIL, KOR and STI. The total number of observations are 1909 since the data collected are in the daily basis mode.

\section{[Insert Table 2 here]}

\subsection{Long and Short - Run Associations using Time Series Analysis (VECM \& Granger Causality)}

Several steps were performed and they include the unit root test, optimal lag selections test as well as cointegration test. In this research, we use augmented Dickey-Fuller (ADF) and the Phillips-Perron (PP) for the test of unit root for Bitcoin and five Asean emerging economies' indices. Table 3 shows the analysis of unit root at level and first difference. The null hypotheses have a unit root which cannot be rejected by both ADF and PP tests. Apparently, after incorporating the first difference, both unit root tests rejected the null hypothesis, concluding that all variables are not stationary at the level basis but it stationary in first difference.

\section{[Insert Table 3 here]}

The identification of lag will be more difficult as lag variables incorporate exogenous variable in the system (Ender, 1995). Thus, for lag selection, we use the shortest lag based on Akaike Information Criteria (AIC). We conducted optimal lag selection for the indices of Asean emerging economies. Referring to Table 4, the Asean countries' index lag chosen are: JPN (lag = 3), KOR (lag = 3), STI (lag = 3), PHIL (lag = 4) and HK (lag = 4).

\section{[Insert Table 4 here]}

Case in hold, we can observe that each of the series is stationary, and therefore, we can evaluate whether there is the presence of long-run equilibrium or vice versa for model test in this study. To assess the existence of long-run association, we refer to Johansen-Juselius Cointegration Tests based on trace and maximum eigenvalue analysis. Again, we analyze the cointegration test using the lag selected in Table 4. As per disclosed in the Table 5, the co-integration result shows that all the Asean emerging economies have co-integration at 1 percent significance level. The trace statistics and maximum eigenvalue postulate the existence of one co-integrating vector, thus confirming that there is a unique co-integrating vector controlling the long-run association between Asean market indices and Bitcoin. These variables are knotted together in the long run, and their eccentricities from the long-run equilibrium path would be rectified. Furthermore, the existence of co-integration also reveals the non-causality among the variables.

[Insert Table 5 here] 
Table 6 shows the long-run association of market indices with bitcoin. All market indices are statistically significant at five percent significance level. Looking at the market index, there seem to be a negative relationship between market indices and bitcoin for JPN, KOR, STI and HK. For example, in the context of JPN, the negative sign of BC coefficient reveals that when BC increases by one percent, it will trigger the decreasing number of JPN market index by $62.63207 \%$. The similar discussion is also applied for other Asean countries which have negative association. A high decrease in market index occurs at KOR where a one percent increase in BC causes a shrink in market index at $117.6253 \%$, followed by $\mathrm{HK}$ and STI markets. A possible reason to this (i.e., increase in bitcoin leads to decrease in market for Korea) is due to the strong Information Technology (IT) infrastructure within the country. Furthermore, the economic slowdown and increase in employment rate in Korea also cause Koreans to look for a better way to invest, especially digitally. On the other hand, we can notice a positive association between BC and PHIL equity market in the long run. The Philippines is considered a good market for bitcoin given the fact that the country has an active bitcoin community and the country has a special economic zone in which foreign crypto exchanges are licensed for operation. Furthermore, the rise of fintech in Philippines also contributes to the rapid development of the bitcoin market in addition to the equity market. This will have a positive long-term economic impact towards Philippines's financial landscape.

\section{[Insert Table 6 here]}

Table 7 discloses information on the Error Correction Term for the market indices of each Asean country. ECT basically looks at the speed of adjustment towards long-run equilibrium. All Asean equity markets obtained negative ECT. For simplicity, Japan is selected as sample for discussion. The ECT of Japan's market Index with BC is -0.006063 , meaning that about six percent of that disequilibrium dissipated before the next period and 94 percent remained. This value is significant at $10 \%$. There may be other factors which this study did not consider to augment or detract from the disequilibrium in the next period.

\section{[Insert Table 7 here]}

We further our investigation to see the dynamic interactions between these variables. In Table 8 , we show the result of the pair wise Granger causality with a lag selected in Table 4 which is enough to whiten the noise process. From Table 7, some general findings can be concluded. Most of the equities index do not have any short-run association with the bitcoin market except for Korea where there is evidence of a short-run association between BC and Korean Index. This supports the idea that bitcoin investment is suitable for long-term investment instead of short-term. Lee et al.'s (2018) study confirms that bitcoin and other cryptocurrencies are better options for investment and diversification in the long-term as correlation between cryptocurrencies is lower as compared to other traditional assets.

\section{[Insert Table 8 here]}

\subsection{Multivariate Generalized Autoregressive Conditional Heteroscedasticity (M-GARCH) - Dynamic Conditional Correlation}

Firstly, we performed the MGARCH-DCC to analyze the association between BC and selected Asean equity markets' index. Basically, the MGARCH-DCC improves the volatility modelling by relaxing some of its assumptions, especially in terms of its means and variances of the factors. We show both GaussianDCC model and t-DCC model. In addition, there are the plotting of estimated conditional correlations and volatilities as well. The main purpose of showing both analyses is to determine model suitability and appropriation to measure the research objective. Table 9 postulates the value of maximum likelihood (ML) prediction of $\lambda_{1}$ and $\lambda_{2}$ (parameters of volatility) and $\delta_{1}$ and $\delta_{2}$ (mean reverting parameters) for each of the return sequences. All the estimated coefficients $\left(\lambda_{1}\right.$ and $\left.\lambda_{2}\right)$ are less than 1 , disclosing that DCCs are following the mean reverting process. This means that BC and stock indexes do not follow I-GARCH and the estimates of $\lambda_{1 \mathrm{i}}, \mathrm{I}=1,2,3,4,5,6$ are close to 1 , implying a steady volatility decay. Furthermore, all 
volatility decay structures are at the significant level. We also present the analysis of t-DCC model to identify the model suitability (see Table 10). The maximum likelihood (ML) of t-DCC postulates that all volatility forecasts are statistically significant and the estimated values are also close to one. Similar to Gaussian-DCC model, these ML estimates for t-DCC also indicate gradual volatility decay. The ML value for t-DCC is 34153.2, higher than 33676.9 and the degree of freedom for t-DCC is 7.4280, less than 30. Given these facts and based on M-GARCH rules, t-DCC model is more suitable and fit for analysis with the series and fat tails. Thus, the discussion of analysis will be based on Table 9. The sum of estimated coefficients $\left(\lambda_{1}\right.$ and $\left.\lambda_{2}\right)$ is less than 1 for most of the preferred parameters. For example, $\lambda_{1}$ JPN $+\lambda 2_{-}$JPN $(0.79256+0.0615=0.9714)$ basically signals that the volatility of JPN Index returns appears to deviate from the IGARCH approach. A similar outcome is also applied for indexes return and shocks to the volatilities for all indexes, which are not tenacious. The lambda totaling of bitcoin $\lambda 1_{-} B C+\lambda 2_{-} B C(0.78431+0.17795$ $=0.96226$ ) still shows that the value is less than 1 , meaning that the volatilities also do not follow IGARCH, thus any shocks to the volatilities are not insistent. Let's say the volatility of any asset is obstinate given the shock in the economy; retail and institutional investors could have a chance of losing all their investment money in the long run although they have made short run profit given the uncertainty level in the bitcoin market. The t-DCC result confirms that the volatilities of BC and Asean stock indexes are not persistent and would significantly alarm investors on whether the investment is safe for them. This outcome is also consistent with a study done by Rahim and Masih (2016).

\section{[Insert Table 9 here]}

[Insert Table 10 here]

Table 11 displays the result of unconditional correlation and volatilities of BC and Asean stock market indexes. The on-diagonal shows unconditional volatilities while off-diagonal indicates the unconditional correlation of each asset. When the unconditional volatility is closer to 0 , the asset thus contains least volatility. However, if the unconditional volatility is close to 1 , the asset encounters higher level of volatility, meaning higher risk. To make it clear, we sort the BC and Asean stock indices from the highest to the lowest volatility (see Table 12). Table 11 illustrates the orders of the unconditional volatilities of the five Asean stock indexes with BC. Interestingly, all the indexes recorded low unconditional volatilities ranging from 0.008534 to 0.014019 . The five stock markets are less volatile whenever there is uncertainty in BC. The BC logged unconditional volatility of 0.070765 which can be considered as a relatively moderate volatile. STI-Singapore index recorded the lowest volatility is. In the news highlighted by DBS Singapore (2018), Singapore investment encounters less volatility as it offers plenty of diversification opportunities to investors.

\section{[Insert Table 11 here]}

\section{[Insert Table 12 here]}

Looking at the off-diagonal components in Table 11, we notice that three stock market indexes have negative correlations, namely, KOR, STI and HK. On the other hand, the other two markets (i.e., JPN and PHIL) have a positive correlation. Results of the unconditional correlation in Table are similar to Table 5 of the long run relationship between $\mathrm{BC}$ and Asean stock indexes. This shows the consistency in our results as predicted by the Vector Error Correction Model and t-DCC model. This is with the exception of one Asean country, where we find a positive correlation (BC and JPN stock index). The Japanese government has legalized (for medium of exchange and investment purposes) the bitcoin currency and the spillover effect has caused the share price to increase. To illustrate, for small exchange listed firms incorporating bitcoin as part of their business transaction, the share price tends to increase as bitcoin prices increase, which coincides with bitcoin's rally (Bitcoin.com, 2017). 
Details of the time framework proportion are given in the footnote.1 As we observe in Figure 1, the co-movements of bitcoin with Asean stock indexes are unpredictable. However, one thing is very clear, and that is bitcoin return is more volatile than stock indexes. During the period of 2010 to 2015, we can see that the volatility was not so explosive, but after 2015, the returns of bitcoin are tremendously volatile. Forbes (2019) reported that bitcoin is still a very speculative asset and most of the time will follow the "Bart Simpson" pattern in which the price would increase, drop, and increase again. The 2013's April meltdown, Fammed 2013 Bubble, bankruptcy of Mt. Gox Bitcoin Exchange, summer sale of 2017, and China's Stern intervention contribute significantly towards these volatiles. A similar sentiment is shared by Cheung et al. (2015) and Corbet et al. (2018c). Comparatively, stock indexes are less volatile than bitcoin as revealed in Figure 1 . The degree of volatilities for bitcoin is higher than the stock indexes during the analysis period in terms of the conditional correlation (see Figure 2). Looking at the unconditional correlation, the degree of relationship can see from different time horizons. During 2010 and 2011, the correlation between BC and HK stock index peaked quite significantly while KOR recorded lowest correlation with BC recording negative value. Other stock indexes observe mixed movement with BC. For example, from 2010 until 2015, there was inconsistent movement but with the same direction, meaning that the correlations co-move together. JPN recorded strong correlation with BC, indicated by the blue color line during the analysis period, followed by PHIL. As these two countries actively promote digital currency and cryptocurrency, this could be the main reason contributing to this movement. KOR, STI, and HK logged inconsistent movement with negative correlation during 2010 until 2019. From 2015 onwards, JPN and BC correlation shows peak level of correlation. However, for STI, the correlation is most of time in the negative-positive, especially during 2012, 2013, 2014, 2016, 2017 and 2019. As mentioned earlier, STI market offers a quite diversified assets platform for investors which influences this movement. The results of Figure 2 and Table 10 (off-diagonal indicates unconditional correlation of each asset) postulate a similar outcome. Thus, given the correlation between stock indexes and BC, we can now classify which markets can be considered a safe investment or safe heaven. Generally, in finance, investors tend to opt for the negative correlation as they can diversify investment risk (Jones, 2014). With this concept, the ones to offer a good investment opportunity (safe zone) in the case of heavy volatility in BC would be STI, HK, and KOR. JPN and PHIL may not be considered as a safe zone because of the strong interdependence which would be very risky for investors. However, this is subject to the investors' attitude towards risk factors.

[Insert Figure 1 here]

[Insert Figure 2 here]

\subsection{Time Frequency Domain using Continuous Wavelet Transform (CWT)}

Before we further discuss the wavelet analysis, we first need to understand their classification prior to making any judgement. Basically, the horizontal line represents the number of years while the vertical line shows the frequency component (scale), with the shorter frequency range closest to the origin of the $\mathrm{BC}$ return fluctuation. Red areas show higher coherency of $\mathrm{BC}$ with stock indexes while blue areas indicate lower co-movement between BC and stock indexes. Additionally, the black solid silhouettes correspond that the co-movement is statistically significant at 5 percent given a particular time and frequency. For example, as we look at the Wavelet Coherence for BC and JPN, the blue color covers a large proportion of

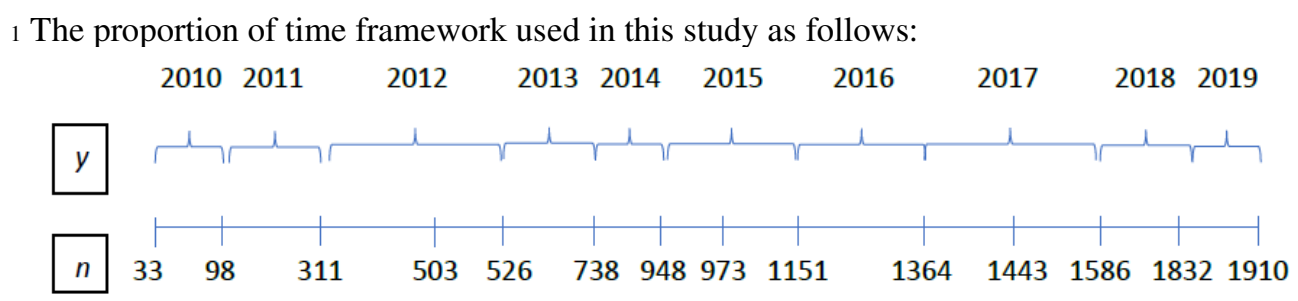


the cone. This means that the time domain co-movement is commonly weak. However, we may notice that the blue area dominates the low scale area (blue color is more prevalent in the higher level of axis y), meaning that the co-movement is more obvious in the long term than it is in the short term. Furthermore, as time progresses, the blue color is less dominant, showing an increase in the co-movement between $\mathrm{BC}$ and JPN. The wavelet phase-difference specifies the dynamic relationship of return or price by looking at the lead-lag association between the paired sets (example: A and B). The arrows set out the lead-lag relationship between the series. If the phase arrow points to the right, this signals that the relationship follows a positive co-movement (in phase: $\mathrm{A}$ and $\mathrm{B}$ ). If the arrow points to the left, the relationship is out of phase (negative co-movement: A and B). If the arrow points down, this implies the price or return of $\mathrm{A}$ leads to the price or return of B. If the arrow points up, then B leads A.

\subsection{CWT for BC with Japan, Korea, Singapore, Philippines and Hong Kong}

As discussed earlier, the CWT reveals the dominance of blue which covers a large proportion of the cone, showing that the co-movement between BC and JPN is relatively low. However, as time progresses, we can observe the increasing dominance of red, meaning that the co-movement is getting stronger (short term, medium and long term). These values are statistically significant at five percent. The outcome is quite pragmatic under the scale of 64 to 256. This result is also consistent with the finding revealed in the MGARCH analysis of the unconditional correlation between BC and JPN stock index which recorded positive correlation. The lead-lag relationship postulates a positive co-movement between BC and JPN stock index where most of the arrows point to the right. In other words, the lead-lad relationship between BC and JPN stock index is within the phase category. If the $\mathrm{BC}$ goes up, then the stock index will also move up. This is consistent with the unconditional correlation shown by our M-GARCH analysis as well (see Figure 3).

The CWT for BC and KOR indicates quite similar results with BC and JPN but for this case, the blue color domain covers the entire cone with little red color areas. This highlights that the degree of comovement is very low even though time progresses. The red color can be observed at the scale of 16 to 64 and 64 to 256 but at minimal level. On the other hand, few arrows are available and most of the arrows point up when looking at the lead-lag relationship, meaning that BC does not lead the KOR stock but the KOR stock index leads the BC. This happens in the medium and long periods. This finding is consistent with the M-GARCH unconditional correlation where the relationship is negative (see Figure 4). Similar to KOR, the outcome for STI is also more or less similar with blue covering most areas in the cone. Just a small proportion of red is available under the scale of 64 to 256. This again shows the degree of comovement which is very low between BC and STI. Again, the wavelet phase difference demonstrates limited arrows that point upward, meaning that the STI stock index leads the BC (see Figure 5). This can be evidenced at scales of 64 to 256 which occur at long term.

In terms of the degree of co-movement between BC and PHIL for short and long scales, it is very clear that blue dominates over red. However, in the medium scale from 16 to 64, red colors some areas. Moreover, the lead-lag relationship shows the arrows pointing to the left. This is an out of phase relationship as the relationship shows a negative co-movement between BC and PHIL during the medium term but not in the short and long periods. Finally, for BC and HK, again blue is dominant, which shows that the comovement between these two remains low. As time progresses, blue remains a dominant color in the cone. Just a small proportion of red color can be observed inside the cone, which only happens at short and medium scale. A limited number of arrows are available inside the cone which can predict the wavelet phase difference. The arrows are available only at the medium scale, such as 16 to 64 . The outcome shows that in the short term, the arrow points to the right while in the long term, the arrow points to the left. Basically, there are in-and-out phases for the lead-lag relationship (see Figure 6 and 7).

[Insert Figure 3 to 7 here]

\section{Conclusion}


The objective of this paper was to investigate the connection between BC with Asean Stock Indexes from 20th July 2010 until 26th April 2019 in terms of long and short run relationships, correlations, volatilities, and time frequency domains as well as lead-lag analysis. The methods used included (i)Vector Error Correction Modeling (VECM) and Granger Causality, (ii) M-GARCH, and (iii) Wavelet Analysis respectively. Firstly, based on VECM, all market indexes showed a long-run association with BC. Four markets recorded negative association and they were JPN, KOR, STI and HK. One market obtained a positive relationship with BC, which was PHIL. Together with that, the Error Correction Term (ECT) postulated a negative speed of adjustment for all Asean markets considering the change in $\mathrm{BC}$ price. We also observed that in the short-run, only one market had a relationship with BC movement (i.e., KOR). This reveals that $\mathrm{BC}$ investment is suitable for long term investment rather than short term considering this aspect. Secondly, further econometrics evidence using M-GARCH analysis revealed that most of the Asean markets recorded low-unconditional volatilities with BC and the only one attained a low value, which was STI. Indirectly, Singapore investment encounters less volatility among other markets. In addition, we also observed that three markets had a negative correlation with BC (i.e., KOR, STI and HK) while two other markets had positive correlation (i.e., JPN and PHIL). These findings are consistent with the prediction made by VECM. Based on the time-frequency domain analysis using the Wavelet, we observed the following outcomes: (i) In the long run, BC and JPN shared strong co-movement while the lead-lag predicted a phase category; (ii) BC, KOR and STI postulated a similar outcome in which the co-movement between the markets was very low and the lead-lag analysis showed an anti-phase relationship; (iii) the comovement between BC and PHIL existed in the medium scale quite significantly but the lead-lag relationship revealed an out-of-phase category; and finally (iv) BC and HK revealed a low co-movement and mixed lead-lag relationship. Our findings have implications for academic and industry practitioners. Theoretically, our study enriches the existing literature available in the area of cryptocurrency, especially as it employs advanced econometrics techniques. Most of the results are consistent and show the different dimensions with regard to long and short run relationships, volatilities, correlations, and time-frequency analysis. We have also utilized Asean emerging economies which are rarely the subject or focus in the literature as existing studies are more skewed towards the West. As for industry practitioners, we believe the outcomes of this study will be a significant sway for retail and institutional investors to design better strategies on diversifying their stock portfolios with different holding period of time horizons and dimensions as mentioned above. Future researchers are encouraged to test these phenomena with other developing countries to see if similar patterns are observable as to that of advanced economies. Comparing Asean countries with developed countries will certainly add value to the existing literature with large data sets.

\section{Declarations}

\section{Ethics approval and consent to participate}

Not applicable.

\section{Consent for publication}

Authors agreed to publish based on Journal of Economic Structures standards.

\section{Availability of data and material}

All data generated or analyzed during this study can be obtained from the corresponding author upon request.

\section{Competing interests}

The authors declare that they have no competing interests.

\section{Funding}


The authors have not received any funding for this study.

\section{Authors' contributions}

Both authors contributed equally to the writing of this paper. Both authors read and approved the final manuscript.

\section{Acknowledgements}

Not applicable.

\section{Authors' information (optional)}

1,2 Department of Economic and Finance, Sunway University Business School, Sunway University, Malaysia.

\section{References}

Bariviera, A. (2017). The inefficiency of bitcoin revisited: a dynamic approach. Economics Letters, 161, 14.

Baur, D. G., Dimpfl, T., \& Kuck, K. (2017). Bitcoin, gold and the us dollar: a replication and extension. Finance Research Letters.

Baur, D. G., Hong, K., \& Lee, A. D. (2017). Bitcoin: Medium of exchange or speculative assets? Journal of International Financial Markets, Institutions and Money.

Blau, B. (2017). Price dynamics and speculative trading in bitcoin. Research in International Business and Finance, 41, 493-499.

Bollerslev, T. (1990). Modelling the coherence in short-run nominal exchange rates: a multivariate generalized ARCH approach. Review of Economics and Statistics, 72, 498-505.

Bouri, E., Jalkh, N., MolnÃąr, P., \& Roubaud, D. (2017). Bitcoin for energy commodities before and after the December 2013 crash: Diversifier, hedge or safe haven? Applied Economics, 49(50), 5063-5073.

Bouri, E., MolnÃąr, P., Azzi, G., Roubaud, D., \& Hagfors, L. (2017). On the hedge and safe haven properties of bitcoin: Is it really more than a diversifier? Finance Research Letters, 20, 192-198.

Bouoiyour, J., Selmi, R., Tiwari, A. K., \& Olayeni, O. R. (2016). What drives Bitcoin price? Economics Bulletin, 36, 843-850.

Brière, M., Oosterlinck, K., \& Szafarz, A. (2015). Virtual currency, tangible return: Portfolio diversification with bitcoin. Journal of Asset Management, 16(6), 365-373.

Charles, A., \& Darn'e, O. (2019). Volatility estimation for bitcoin: Replication and robustness. International Economics.

Cheah, E.-T., \& Fry, J. (2015). Speculative bubbles in bitcoin markets? An empirical investigation into the fundamental value of bitcoin. Economics Letters, 130, 32-36.

Cheung A., Roca, E., \& Su, J. (2013). Crypto-currency bubbles: an application of the Phillips Shi-Yu methodology on Mt. Gox Bitcoin prices. Applied Economics, 47(23), 2348-2358.

Conrad, C., Custovic, A., \& Ghysels, E. (2018). Long-and short-term cryptocurrency volatility components: a GARCH-MIDAS analysis. Journal of Risk and Financial Management, 11(2), 23.

Corbet, S., Larkin, C., Lucey, B., Meegan, A., \& Yarovaya, L. (2018a). Exploring the dynamic relationships between cryptocurrencies and other financial assets. Economics Letters, 165(1), 28-34.

Corbet, S., Larkin, C., Lucey, B., Meegan, A., \& Yarovaya, L. (2018b). The volatility generating effects of macroeconomic news on cryptocurrency returns. Available at https://ssrn.com/abstract_id=3141986.

Dwyer, G. (2015). The economics of bitcoin and similar private digital currencies. Journal of Financial Stability, 17, 81-91.

Dyhrberg, A. H. (2016). Bitcoin, gold and the dollar: a GARCH volatility analysis. Finance Research Letters, 16, 85-92.

Ender, M. G (1995). The use of telecommunications by the soldiers of Operation Just Cause. Armed Forces Society, 21, 435-453. 
Engle, R. F. (2002). Dynamic conditional correlation: a simple class of multivariate GARCH models. Journal of Business and Economic Statistics, 20, 339-350.

Engle, R. F., \& Sheppard, K., (2001). Theoretical and empirical properties of dynamic conditional correlation multivariate GARCH. NBER Working Paper, 8554.

Forbes (2019). URL: https://www.forbes.com/ . Accessed on 24/2/2020.

Guesmi, Y., Agougui, H., Lafi, R., et al. (2018). Synthesis of hydroxyapatite-sodium alginate via a coprecipitation technique for efficient adsorption of methylene blue dye. Journal of Molecular Liquids, 249, 912-920.

Jones, L. (2014). Examining the forensic artifacts produced by the use of Bitcoin currency. ProQuest database.

Katsiampa, P. (2017). Volatility estimation for bitcoin: a comparison of GARCH models. Economics Letters, 158, 3-6.

Kristoufek, L. (2013). Bitcoin meets Google Trends and Wikipedia: Quantifying the relationship between phenomena of the Internet era. Scientific Reports, 3, 3415.

Lee, D. K. C., Guo, L., \& Wang, Y. (2018). Cryptocurrency: a new investment opportunity? The Journal of Alternative Investments, 20(3), 16-40.

Nadarajah, S., \& Chu, J. (2017). On the inefficiency of bitcoin. Economics Letters, 150, 6-9.

Nakamoto, S. (2008). Bitcoin: A Peer-to-Peer Electronic Cash System. https://bitcoin.org/bitcoin.pdf

Namecoin. (2014) Namecoin. https://www.namecoin.org/

Platanakis, E., \& Urquhart, A. (2018). Should investors include bitcoin in their portfolios? A portfolio theory approach. In Cambridge Cryptocurrency Research Conference (1-26).

Polasik, M., Piotrowska, A., Wisniewski, T. P., Kotkowski, R., \& Lightfoot, G. (2015). Price fluctuations and the use of bitcoin: an empirical inquiry. International Journal of Electronic Commerce, 20(1), 9-49.

Rahim, A. M., \& Masih, M. (2016). Portfolio diversification benefits of Islamic investors with their major trading partners: Evidence from Malaysia based on MGARCH-DCC and wavelet approaches. Economic Modelling, 54, 425-438.

Rua, A., \& Nunes, L. C. (2009). International co-movement of stock market returns: a wavelet analysis. Journal of Empirical Finance, 16(4), 632-639.

Shen, D., Urquhart, A., \& Wang, P. (2019). Does twitter predict bitcoin? Economics Letters, 174, 118-122.

Urquhart, A. (2016). The inefficiency of bitcoin. Economics Letters, 148, 80-82.

Urquhart, A. (2017). Price clustering in Bitcoin. Economics Letters, 159, 145-148.

Urquhart, A., \& Zhang, H. (2019). Is Bitcoin a hedge or safe haven for currencies? An intraday analysis. International Review of Financial Analysis, 63, 49-57.

Walther, T., Klein, T., Pham Thu, H., \& Piontek, K. (2017). True or spurious long memory in European Non-EMU currencies. Research in International Business and Finance, 40, 217-230.

Weber, B. (2014). Bitcoin and the legitimacy crisis of money. Cambridge Journal of Economics, 40(1), 1741.

Yi, S., Xu, Z., \& Wang, G.-J. (2018). Volatility connectedness in the cryptocurrency market: Is Bitcoin a dominant cryptocurrency? International Review of Financial Analysis, 60, 98-114. 


\section{Appendix}

Table 1: Description of Variables

\begin{tabular}{lll}
\hline No & Symbol & Definition \\
\hline 1 & BC & Bitcoin Index \\
2 & JPN & Nikkei 225 Index - Japan \\
3 & KOR & KOSPI - Korea \\
4 & STI & FTSE Straits Times Index - Singapore \\
5 & PHIL & PSEi Index - Philippines \\
6 & HK & Hang Seng Index - Hong Kong \\
\hline
\end{tabular}

Note: This table shows further details on the variables chosen in this study. Overall, we have chosen five Asean countries for analysis purposes.

Table 2: Descriptive Statistics

\begin{tabular}{ccccccc}
\hline & BC & JPN & KOR & STI & PHIL & HK \\
\hline Mean & 0.005874 & 0.000458 & 0.000119 & $6.79 \mathrm{E}-05$ & 0.000439 & 0.000199 \\
Maximum & 0.515155 & 0.074262 & 0.055307 & 0.048676 & 0.055419 & 0.055187 \\
Minimum & -0.470004 & -0.111534 & -0.085676 & -0.056870 & -0.095984 & -0.080260 \\
Std. Dev. & 0.070157 & 0.013955 & 0.010066 & 0.008487 & 0.011323 & 0.012315 \\
Obs & 1909 & 1909 & 1909 & 1909 & 1909 & 1909 \\
\hline
\end{tabular}

Note: This table illustrates the descriptive statistics of the data used in this study. It consists five elements (i.e., mean, $\mathrm{SD}=$ standard deviation, $\mathrm{Min}=$ minimum value, $\mathrm{Max}=$ maximum value and $\mathrm{Obs}=\mathrm{Observation}$ ). The samples are Bitcoin return and Asean Economies Stock Indexes Return.

Table 3: Unit Root Tests (ADF and PP Unit Root Tests) for Bitcoin and Equity Indices

\begin{tabular}{ccccc}
\hline Variables & \multicolumn{2}{c}{ Level } & \multicolumn{2}{c}{ First Difference } \\
\cline { 2 - 5 } & ADF & PP & ADF & PP \\
\hline BC & -2.403547 & -2.501709 & $-14.19600^{*}$ & $-43.72934^{*}$ \\
JPN & -2.863470 & -2.779592 & $-45.77698^{*}$ & $-45.80180^{*}$ \\
KOR & -3.182944 & -3.076102 & $-43.00685^{* *}$ & $-43.06836^{*}$ \\
STI & -2.716996 & -2.892518 & $-42.40451^{*}$ & $-42.42439^{*}$ \\
PHIL & -2.418378 & -2.277534 & $-43.22455^{*}$ & $-43.36255^{*}$ \\
HK & -2.703887 & -2.750872 & $-43.04464^{*}$ & $-43.04038^{*}$ \\
\hline
\end{tabular}

Note: $* * *, * * *$ indicate significance at $1 \%, 5 \%, 10 \%$ respectively.

Table 4: VAR Lag Order Selection Criteria for Equity Indices

\begin{tabular}{cccccc}
\hline & JPN & KOR & STI & PHIL & HK \\
\hline $\begin{array}{c}\text { Akaike Information Criterion } \\
\text { (AIC) }\end{array}$ & -8.186374 & -8.844965 & -9.183065 & -8.603191 & -8.434908 \\
Lags & 3 & 3 & 3 & & \\
\hline
\end{tabular}


Table 5: Johansen-Juselius Cointegration Tests for Equity Indices

\begin{tabular}{cccc}
\hline \multirow{2}{*}{ Markets } & Null Hypothesis & \multicolumn{2}{c}{ T- Statistics (5\%) } \\
\cline { 3 - 4 } & & Trace & Max Eigen \\
\hline JPN $^{*}$ & At Most One & 803.3344 & 345.2216 \\
KOR $^{*}$ & At Most One & 808.5560 & 465.6239 \\
STI $^{*}$ & At Most One & 762.3281 & 423.3376 \\
PHIL $^{*}$ & At Most One & 694.4797 & 409.5602 \\
HK$^{*}$ & At Most One & 630.5607 & 346.9450 \\
\hline
\end{tabular}

Note: $*, * * * * *$ indicate significance at $1 \%, 5 \%, 10 \%$ respectively.

Table 6: Long-Run Relationship of Market Indices and Bitcoin

\begin{tabular}{cc}
\hline Countries & Asean Markets Index \\
\hline JPN & JPN $=0.023181-62.63207 \mathrm{BC}$ \\
KOR & {$[-22.7723]$} \\
& KOR $=0.009627-117.6253 \mathrm{BC}$ \\
STI & {$[-22.8851]$} \\
& STI $=-0.003898-27.15635 \mathrm{BC}$ \\
PHIL & {$[-20.9501]$} \\
& PHIL $=-0.069235+145.7809 \mathrm{BC}$ \\
HK & {$[21.3017]$} \\
& HK $=0.002984-43.45732 \mathrm{BC}$ \\
\end{tabular}

Table 7: Error Correction Term (ECT) of Market Indices with Bitcoin

\begin{tabular}{cc}
\hline Countries & ECT \\
\hline JPN & $-0.006063 *$ \\
KOR & $-0.004232^{*}$ \\
STI & $-0.096677^{*}$ \\
PHIL & $-0.000736^{* *}$ \\
HK & $-0.023501 *$ \\
\hline Note: $* * *, * * *$ indicate significance at $1 \%, 5 \%, 10 \%$ respectively.
\end{tabular}

Table 8: Short-Run Granger Causality

\begin{tabular}{|c|c|c|c|}
\hline Countries & Null Hypothesis & F-Statistics & Prob \\
\hline JPN & JPN does not Granger Cause BC & 0.80178 & 0.4928 \\
\hline & BC does not Granger Cause JPN & 0.51775 & 0.6701 \\
\hline KOR & KOR does not Granger Cause BC & 1.39119 & 0.2437 \\
\hline & BC does not Granger Cause KOR & 2.14055 & $0.0932^{* * *}$ \\
\hline STI & STI does not Granger Cause BC & 2.15483 & $0.0914^{* * *}$ \\
\hline & BC does not Granger Cause STI & 1.17690 & 0.3171 \\
\hline PHIL & PHIL does not Granger Cause BC & 0.22794 & 0.9228 \\
\hline & BC does not Granger Cause PHIL & 0.82782 & 0.5073 \\
\hline HK & HK does not Granger Cause BC & 2.20766 & $0.0659^{* * *}$ \\
\hline & BC does not Granger Cause HK & 0.73138 & 0.5705 \\
\hline
\end{tabular}

Note: $*, * *, * * *$ indicate significance at $1 \%, 5 \%, 10 \%$ respectively 
Table 9: Maximum likelihood estimates of the Gaussian-DCC model

\begin{tabular}{lcccc}
\hline Parameter & Estimate & SE & t- Ratio & {$[$ Prob] } \\
\hline Lambdal $(\lambda I)$ & & & & \\
BC & 0.81000 & 0.023547 & 34.3989 & 0.000 \\
JPN & 0.81131 & 0.034595 & 23.4514 & 0.000 \\
KOR & 0.86167 & 0.025027 & 34.4303 & 0.000 \\
STI & 0.93161 & 0.013535 & 68.8290 & 0.000 \\
PHIL & 0.76420 & 0.043385 & 17.6144 & 0.000 \\
HK & 0.94599 & 0.009431 & 100.3046 & 0.000 \\
Lambda2 $(\lambda 2)$ & & & & \\
BC & 0.14338 & 0.014886 & 9.6321 & 0.000 \\
JPN & 0.09972 & 0.015097 & 6.6055 & 0.000 \\
KOR & 0.06829 & 0.011193 & 6.1014 & 0.000 \\
STI & 0.04358 & 0.007429 & 5.8664 & 0.000 \\
PHIL & 0.13110 & 0.019541 & 6.7091 & 0.000 \\
HK & 0.03284 & 0.004814 & 6.8221 & 0.000 \\
Maximized log-likelihood & 33676.9 & & & \\
df & & & & \\
\hline
\end{tabular}

Note: This table reveals statistics of maximum likelihood based on Gaussian-DCC model for Bitcoin and Asean indices. $\lambda 1$ and $\lambda 2$ are decay factors for variance and covariance, respectively.

Table 10: Maximum likelihood estimates of the t-DCC model

\begin{tabular}{lcccc}
\hline Parameter & Estimate & SE & t- Ratio & {$[$ Prob] } \\
\hline Lambdal $(\lambda 1)$ & & & & \\
BC & 0.78431 & 0.022993 & 34.1113 & 0.000 \\
JPN & 0.79256 & 0.039047 & 20.2976 & 0.000 \\
KOR & 0.91799 & 0.020504 & 44.7711 & 0.000 \\
STI & 0.92237 & 0.018229 & 50.5984 & 0.000 \\
PHIL & 0.82413 & 0.041149 & 20.0280 & 0.000 \\
HK & 0.94304 & 0.012236 & 77.0736 & 0.000 \\
& & & & \\
Lambda2 $(\lambda 2)$ & & & & \\
BC & 0.17795 & 0.017206 & 10.3420 & 0.000 \\
JPN & 0.10090 & 0.017102 & 5.9002 & 0.000 \\
KOR & 0.04696 & 0.009756 & 4.8137 & 0.000 \\
STI & 0.04475 & 0.009026 & 4.9587 & 0.000 \\
PHIL & 0.09367 & 0.018557 & 5.0478 & 0.000 \\
HK & 0.03017 & 0.005528 & 5.4570 & 0.000 \\
Maximized log-likelihood & 34153.2 & & & \\
df & 7.4280 & & & \\
\hline
\end{tabular}

Note: This table reveals statistics of maximum likelihood based on t-DCC model for Bitcoin and Asean indices. $\lambda 1$ and $\lambda 2$ are decay factors for variance and covariance, respectively. 
Table 11: Unconditional correlation and volatility (based on t-DCC model)

\begin{tabular}{lcccccc}
\hline \multicolumn{1}{c}{ Parameters } & BC & JPN & KOR & STI & PHIL & HK \\
\hline BC & 0.070765 & & & & & \\
JPN & 0.036582 & 0.014019 & & & & \\
KOR & -0.009961 & 0.133520 & 0.010106 & & & \\
STI & -0.027420 & 0.201950 & 0.576820 & 0.008534 & & \\
PHIL & 0.009212 & 0.067884 & 0.424900 & 0.418810 & 0.011383 & \\
HK & -0.018871 & 0.157810 & 0.622470 & 0.710420 & 0.417870 & 0.012370 \\
\hline
\end{tabular}

Note: This table shows the unconditional correlation and volatility for Bitcoin and Asean indices based on t-DCC model. The time horizon used in this study was from July 20, 2010 until April 26, 2019 (daily basis data). It involves five Asean countries.

Table 12: The ranks of unconditional volatilities of Bitcoin and Asean Indices

\begin{tabular}{ccc}
\hline No. & BC and Asean Indices & Unconditional Volatility \\
\hline 1 & BC & 0.070765 \\
2 & JPN & 0.014019 \\
3 & HK & 0.012370 \\
4 & PHIL & 0.011383 \\
5 & KOR & 0.010106 \\
6 & STI & 0.008534 \\
\hline
\end{tabular}

Note: This table shows the rank of unconditional volatilities for Bitcoin and Asean indices based on t-DCC model. The time horizon used in this study was from July 20, 2010 until April 26, 2019 (daily basis data). It involves five Asean countries.

Plot of conditional volatilities and correlations

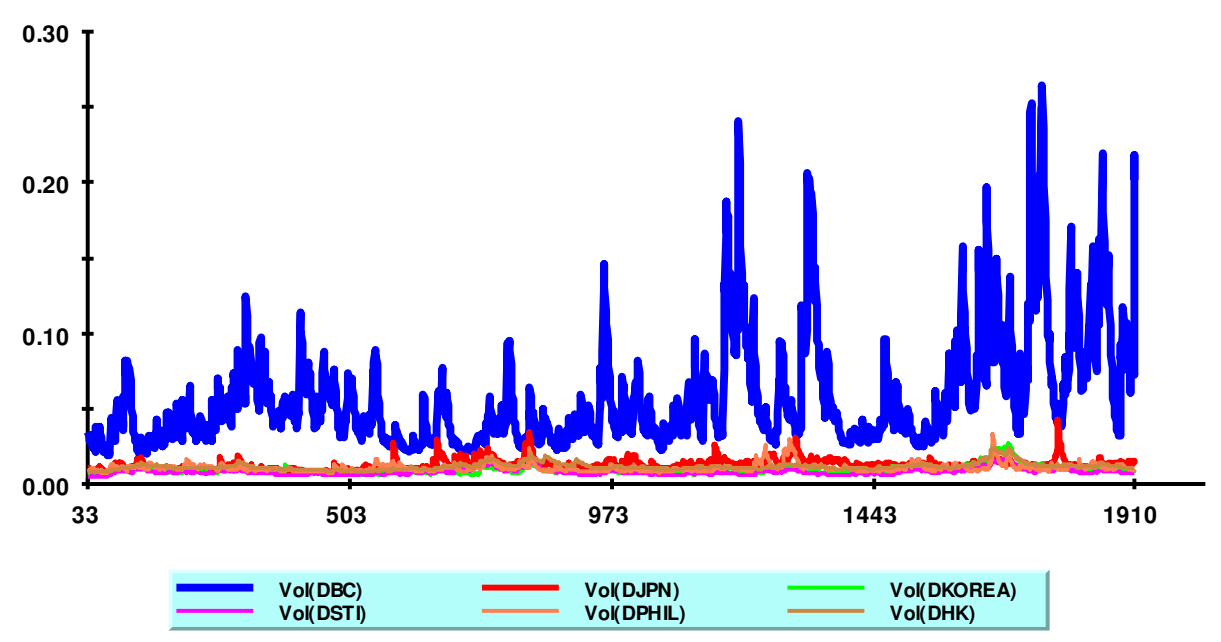

Figure 1. Conditional volatilities of Bitcoin with Asean Indices 
Plot of conditional volatilities and correlations

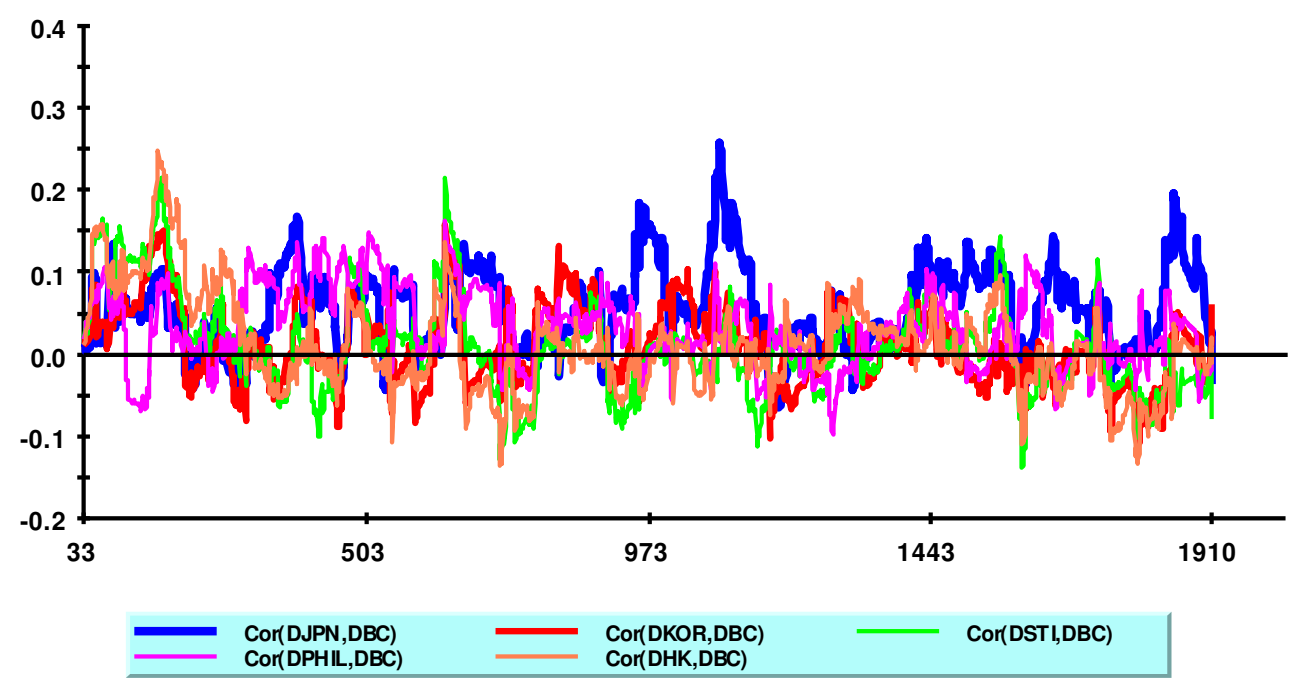

Figure 2: Conditional correlations of Bitcoin with Asean Indices

Wavelet Coherence: RBC vs RJPN

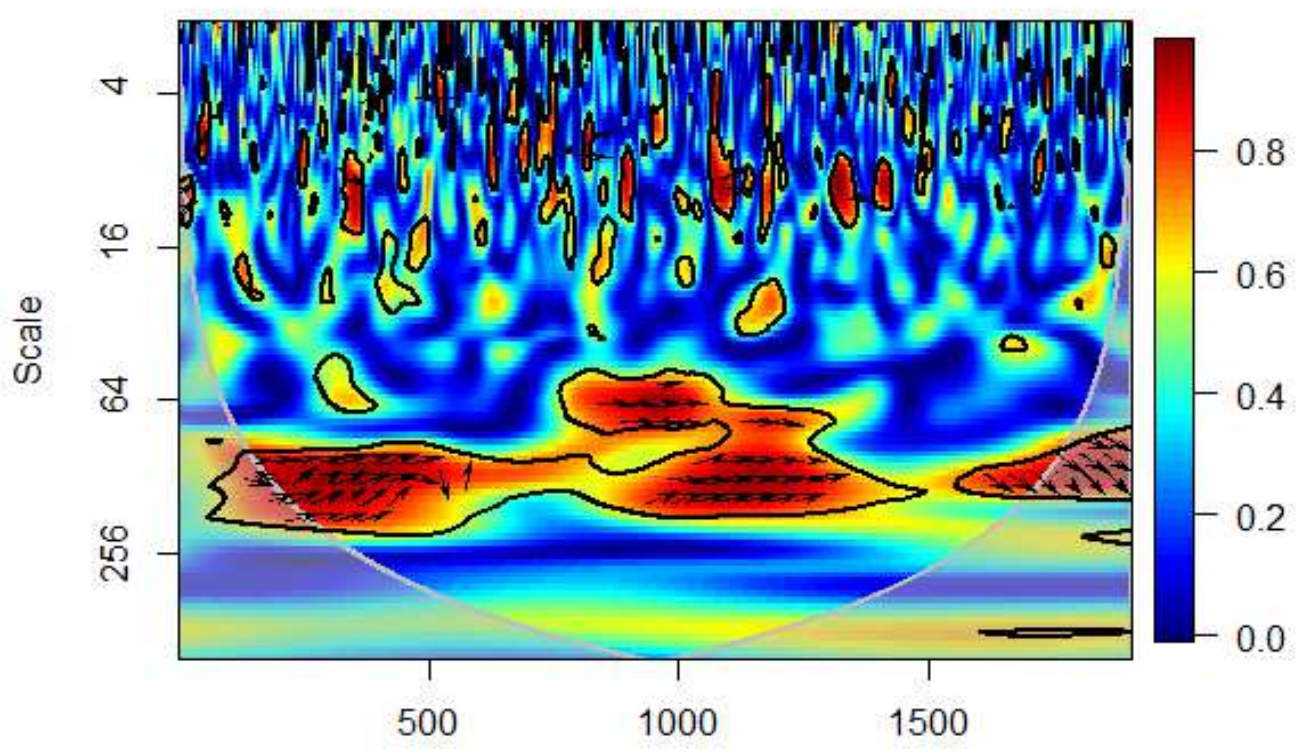

Period

Figure 3: Continuous Wavelet Transform (CWT) for BC and JPN Stock Index 


\section{Wavelet Coherence: RBC vs RKOREA}

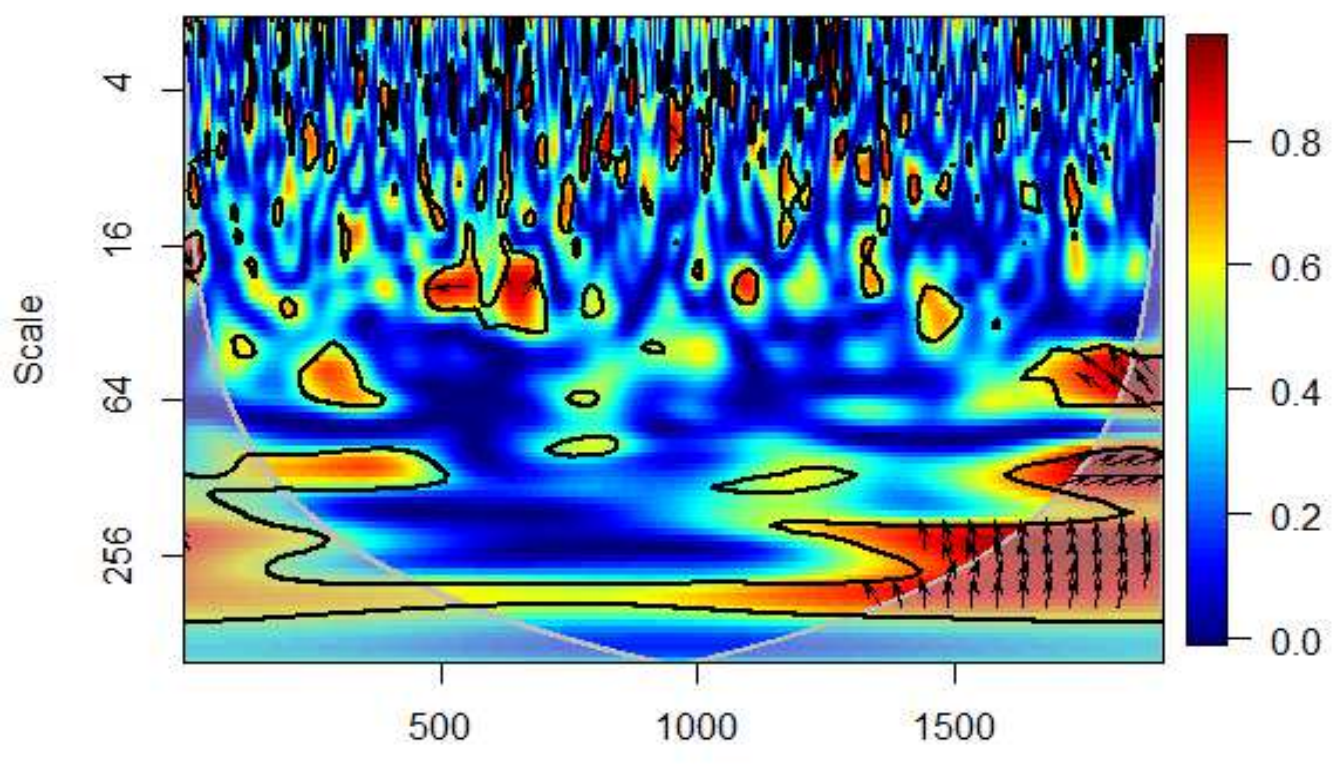

Period

Figure 4: Continuous Wavelet Transform (CWT) for BC and Korea Stock Index

\section{Wavelet Coherence: RBC vs RSTI}

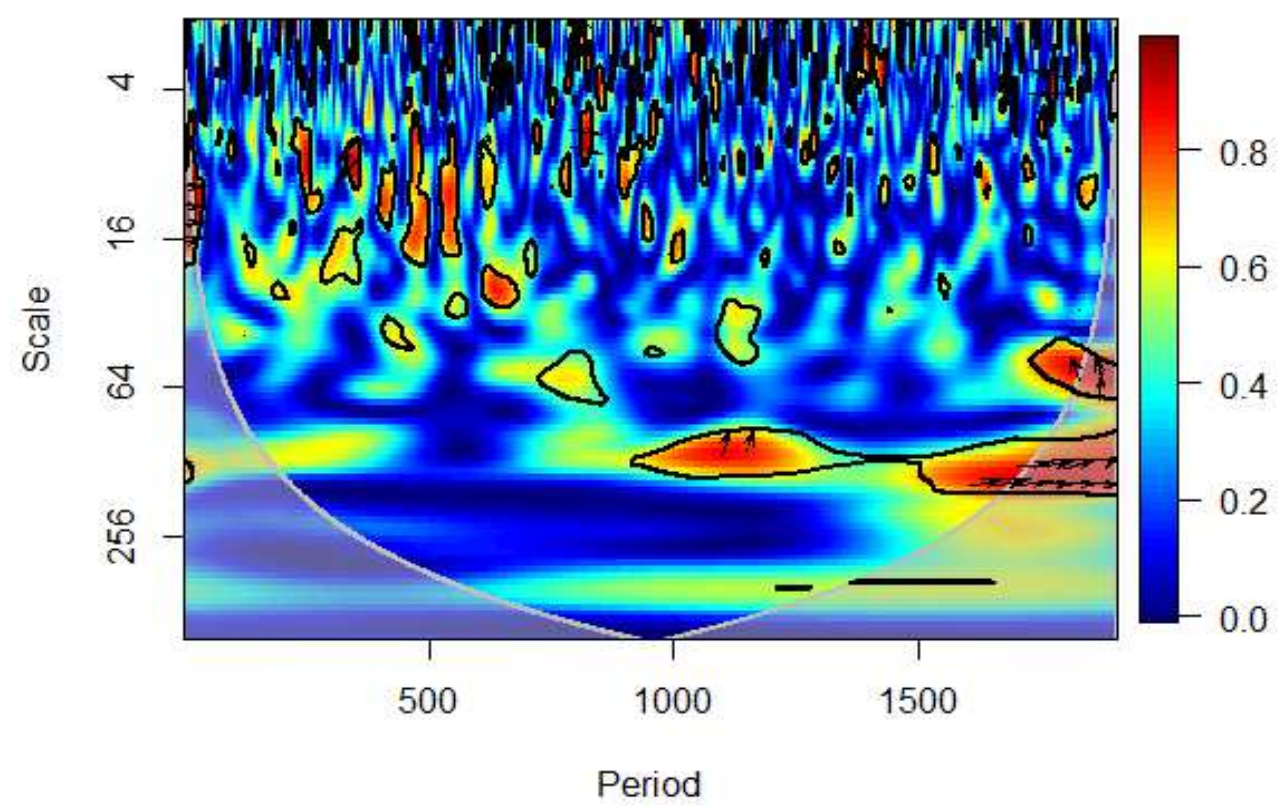

Figure 5: Continuous Wavelet Transform (CWT) for BC and Singapore Stock Index 
Wavelet Coherence: RBC vs RPHIL

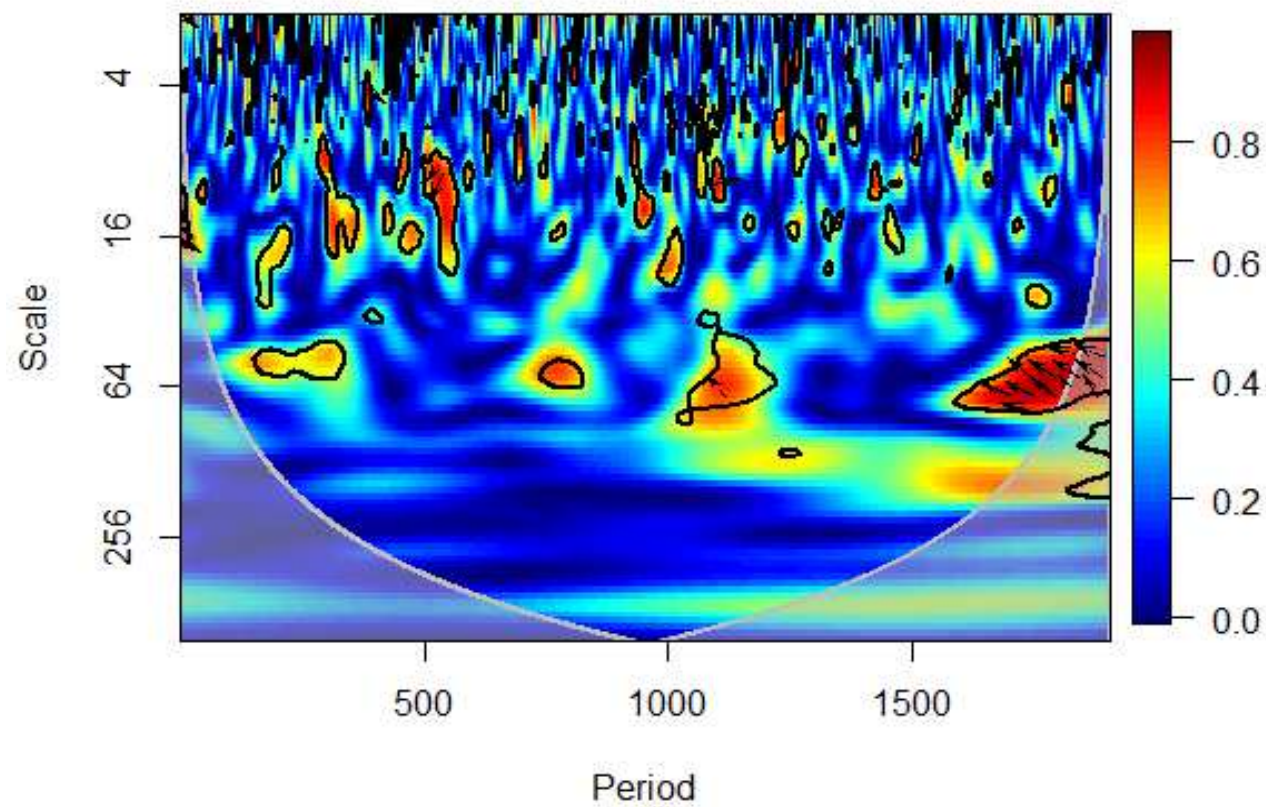

Figure 6: Continuous Wavelet Transform (CWT) for BC and Philippines Stock Index

Wavelet Coherence: RBC vs RHK

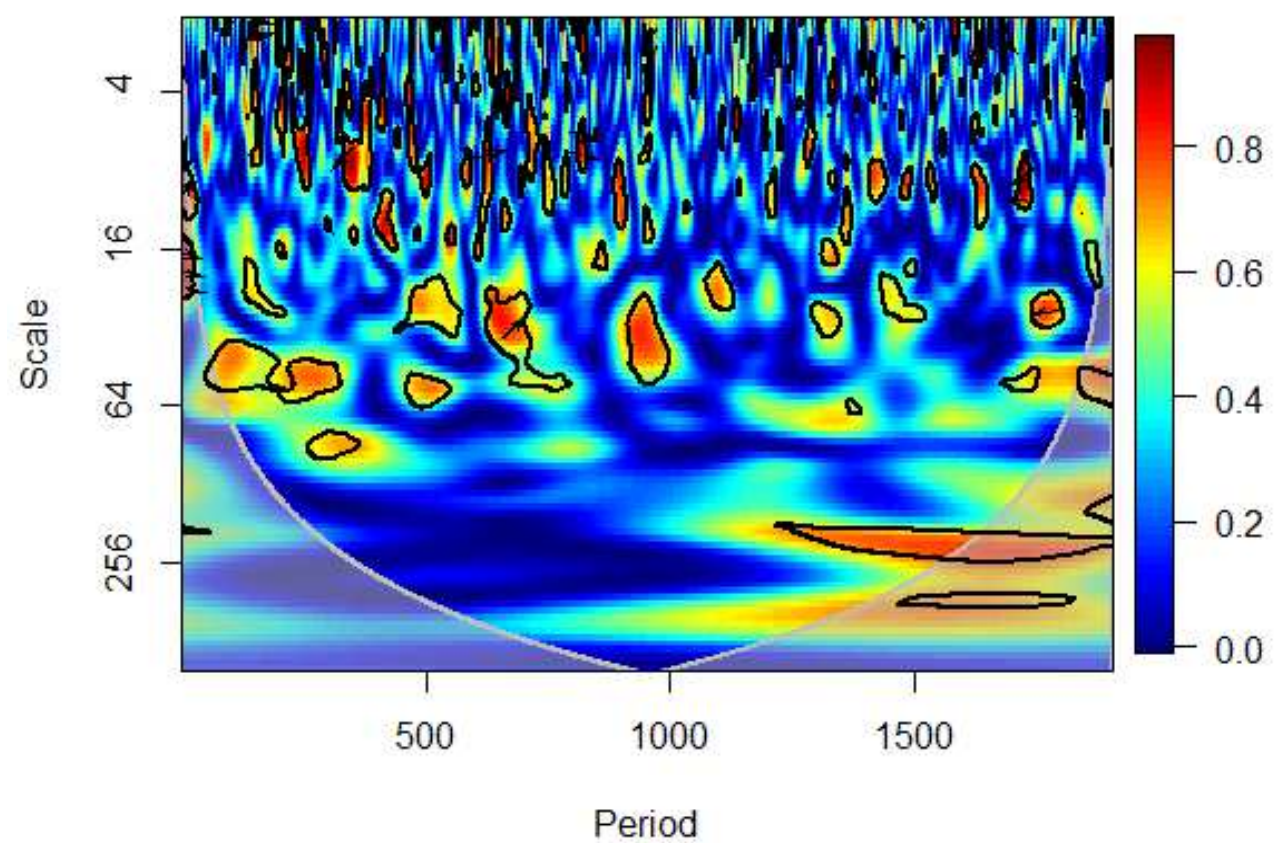

Figure 7: Continuous Wavelet Transform (CWT) for BC and Hong Kong Stock Index 